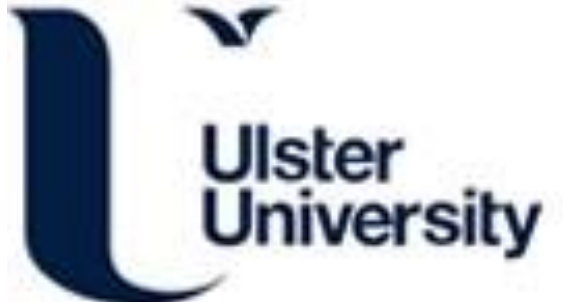

\section{Foundations and interpretations of the pulsed-Townsend experiment}

Casey, M., Stokes, P., Cocks, D. G., Bošnjaković, D., Simonović, I., Brunger, M., Dujko, S., Petrović, Z., Robson, R., \& White, R. (2021). Foundations and interpretations of the pulsed-Townsend experiment. Plasma Sources Science and Technology, 30(3), 035017. [ 035017]. https://doi.org/10.1088/1361-6595/abe729

Link to publication record in Ulster University Research Portal

\section{Published in:}

Plasma Sources Science and Technology

Publication Status:

Published (in print/issue): 01/03/2021

DOI:

10.1088/1361-6595/abe729

\section{Document Version}

Publisher's PDF, also known as Version of record

\section{General rights}

Copyright for the publications made accessible via Ulster University's Research Portal is retained by the author(s) and / or other copyright owners and it is a condition of accessing these publications that users recognise and abide by the legal requirements associated with these rights.

\section{Take down policy}

The Research Portal is Ulster University's institutional repository that provides access to Ulster's research outputs. Every effort has been made to ensure that content in the Research Portal does not infringe any person's rights, or applicable UK laws. If you discover content in the Research Portal that you believe breaches copyright or violates any law, please contact pure-support@ulster.ac.uk. 
PAPER • OPEN ACCESS

\section{Foundations and interpretations of the pulsed-Townsend experiment}

To cite this article: M J E Casey et al 2021 Plasma Sources Sci. Technol. 30035017

View the article online for updates and enhancements.

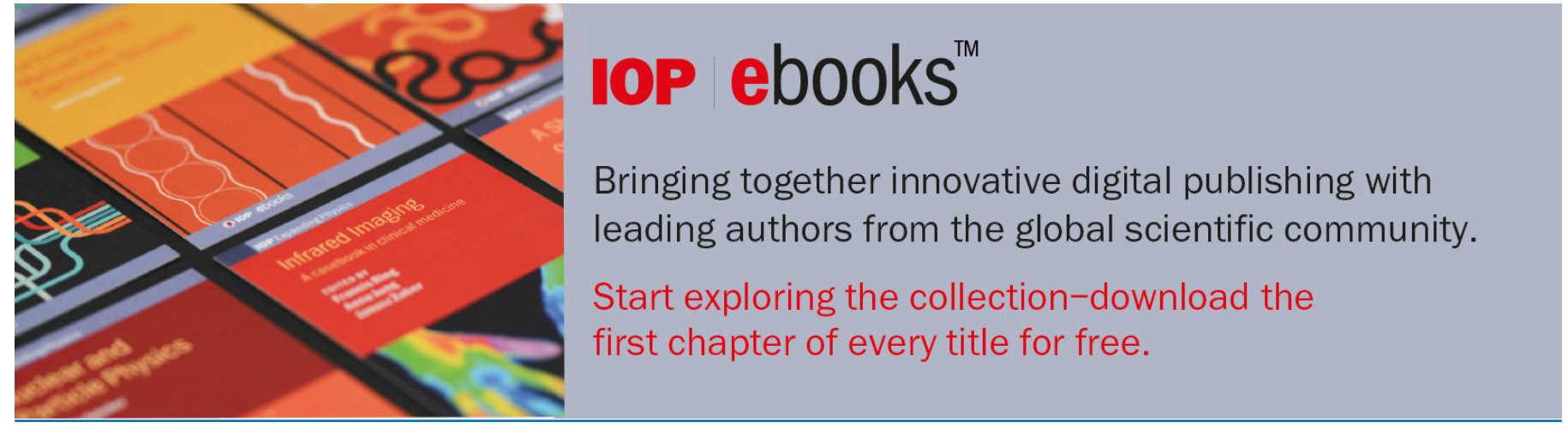

This content was downloaded from IP address 137.219.201.135 on 19/04/2021 at 01:23 


\title{
Foundations and interpretations of the pulsed-Townsend experiment
}

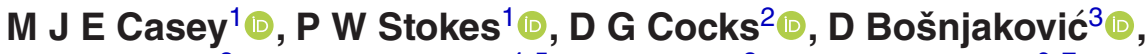

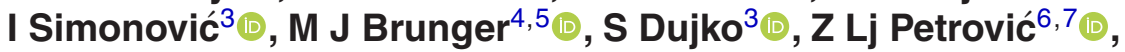 \\ R E Robson ${ }^{1,8}$ (i) and R D White ${ }^{1, *}$ (i) \\ ${ }^{1}$ College of Science and Engineering, James Cook University, Townsville, QLD, Australia \\ 2 Research School of Physics, The Australian National University, Canberra, ACT, Australia \\ ${ }^{3}$ Institute of Physics, University of Belgrade, Zemun, Belgrade, Serbia \\ ${ }^{4}$ College of Science and Engineering, Flinders University, Adelaide, SA, Australia \\ 5 Department of Actuarial Science and Applied Statistics, Faculty of Business and Information Science, \\ UCSI University, Kuala Lumpur, Malaysia \\ 6 School of Engineering, Ulster University, Newtownabbey, Antrim, Northern Ireland, United Kingdom \\ ${ }^{7}$ Serbian Academy of Sciences and Arts, Belgrade, Serbia \\ ${ }^{8}$ Centre for Quantum Dynamics, Griffith University, Nathan, QLD, Australia \\ E-mail: ronald.white@jcu.edu.au
}

Received 22 November 2020, revised 1 February 2021

Accepted for publication 17 February 2021

Published 25 March 2021

\begin{abstract}
The pulsed-Townsend (PT) experiment is a well known swarm technique used to measure transport properties from a current in an external circuit, the analysis of which is based on the governing equation of continuity. In this paper, the Brambring representation (1964 Z. Phys. 179 532) of the equation of continuity often used to analyse the PT experiment, is shown to be fundamentally flawed when non-conservative processes are operative. The Brambring representation of the continuity equation is not derivable from Boltzmann's equation and consequently transport properties defined within the framework are not clearly representable in terms of the phase-space distribution function. We present a re-analysis of the PT experiment in terms of the standard diffusion equation which has firm kinetic theory foundations, furnishing an expression for the current measured by the PT experiment in terms of the universal bulk transport coefficients (net ionisation rate, bulk drift velocity and bulk longitudinal diffusion coefficient). Furthermore, a relationship between the transport properties previously extracted from the PT experiment using the Brambring representation, and the universal bulk transport coefficients is presented. The validity of the relationship is tested for two gases Ar and $\mathrm{SF}_{6}$, highlighting also estimates of the differences.
\end{abstract}

Keywords: pulsed townsend experiment, transport coefficient definition, pulsed townsend governing equation, kinetic theory, Brambring's equation

(Some figures may appear in colour only in the online journal)

\section{Introduction}

The use of accurate electron swarm transport coefficients in simulations has wide ranging implications for modelling

* Author to whom any correspondence should be addressed.

(c) (i) Original content from this work may be used under the terms of the Creative Commons Attribution 4.0 licence. Any further distribution of this work must maintain attribution to the author(s) and the title of the work, journal citation and DOI. physics, from atmospheric processes through to medical imaging and therapies [2-21]. For the well established swarm experimental techniques, the various experimental parameters (such as temperature, sample purity, uniformity of the applied field, ...) are assumed to be highly accurate (within the reported error bars), and the techniques for extracting the measured quantities are generally considered to be well understood. Within the swarm community itself, consensus on the 
extraction of transport parameters/coefficients is essential as this explicitly impacts upon the accuracy of swarm-derived cross-sections [22-43] that may be subsequently used directly for modelling of gas and liquid-phase transport $[12,13]$, or the direct application of electron swarm transport coefficients in fluid modelling of plasmas [3, 44]. As such, high accuracy is required in both the measurement and definition of the transport coefficients to ensure applications in technology and medicine can be made with confidence.

Transport coefficient definition/measurement was an active area of debate in the 1960-1990s [45-54], and misunderstandings still exist [30]. For example, it is important to understand that different swarm experiments operate in different regimes - time of flight (TOF) and pulsed-Townsend (PT) for example in the hydrodynamic regime, where the space-time dependence of all quantities can be projected onto the number density, $n(\mathbf{r}, t)$ [55], while the steady state Townsend (SST) approach operates in the non-hydrodynamic regime, where one has to treat the space $(\mathbf{r})$ and time $(t)$ dependence more generally $[47,56]$.

In the hydrodynamic regime, there are two fundamental types of transport coefficients, which we call flux and bulk ${ }^{10}$. The flux coefficients are defined through well-known fluxgradient relationships, such as Fick's law. The bulk coefficients, however, are defined through the diffusion equation, which applies, for example, to the analysis of the various measured currents in both the TOF and PT experiments. Thus we can say quite generally, without reference to the specific form of the solution of the diffusion equation, for any experiment amenable to a hydrodynamic description, that it is the bulk quantities which are extracted and therefore it is these which are tabulated in the literature. On the other hand, the SST experiment is inherently non-hydrodynamic, and measures the microscopic Townsend ionisation coefficient, $\alpha$, through the particle density relation $n \sim \exp (\alpha z)$. The SST experiment cannot be analysed through the diffusion equation [57], and therefore does not measure any of the hydrodynamic transport coefficients.

In spite of much discussion over the past 30-60 years [45-54], there does, however, remain some residual confusion about what transport coefficients/properties are extracted from the PT experiment, and how they relate to the standard flux and bulk transport coefficients ${ }^{11}$. Currently, the PT experiment is one of the swarm methods in active use, with key groups in Switzerland [58, 63] and México [32, 68], as well as the scanning drift tube experiment in Hungary [69-71] and the doubleshutter drift tube experiment in Japan [72], which provide much of the present-day electron swarm data. Consequently, it is essential that the transport properties extracted from the PT

\footnotetext{
${ }^{10}$ While some associate them with particular experiments, such nomenclature hides their fundamental nature [50].

${ }^{11}$ With regard to the extracted coefficients, we note that while some PT analyses report $\alpha_{\mathrm{T}} / n_{0}$ (the macroscopic form of Townsend's first ionisation coefficient), the quantity $R_{\text {net }}$ has been extracted directly by Franck and co-workers [58-64] and Ridenti et al [65], reported for the PT measurements of Aschwanden [66] (along with $\alpha_{\mathrm{T}} / n_{0}$ ) when analysis of the current transients was hindered by the strong electron attachment, and in Phelps and Pitchford [67] measurements were transformed to $R_{\text {net }}$, to illustrate a few examples.
}

experiments are identified correctly. This represents the focus of the current study.

We begin this paper with a brief review of fundamental swarm transport theory and definitions in section 2 , in order to revisit the vexed issue of transport coefficient definition in relation to the PT experiment and their relation to transport coefficients which are derivable from the Boltzmann equation. We demonstrate that the Brambring form of the continuity/diffusion equation [1] generally used to analyse the PT experiment is fundamentally flawed when non-conservative processes are operative. A general solution of the full diffusion equation has long been available in the literature [73, 74], and in section 3 we specifically show how it can be adapted to the PT experiment to extract the standard definitions of the transport coefficients with firm foundations in kinetic theory. With our focus on the PT experiment, in section 3 we demonstrate that the existing transport property measurements extracted from PT experiments using the fundamentally flawed Brambring equation for the current in the external circuit [1], can be transformed to the standard bulk transport coefficient definitions. Transformation of existing measured PT transport properties, for the particular examples of $\mathrm{Ar}$ and $\mathrm{SF}_{6}$, are presented in section 4 and compared with the bulk transport coefficients extracted from TOF experiments and calculated using a multi-term solution of Boltzmann's equation. Thereafter, some concluding remarks are drawn in section 5.

\section{Theory}

\subsection{The exact continuity equation, the hydrodynamic regime and the diffusion equation}

The exact continuity equation can be derived either from first principles, or from Boltzmann's equation:

$$
\frac{\partial \mathrm{f}}{\partial \mathrm{t}}+\boldsymbol{v} \cdot \nabla f+\mathbf{a} \cdot \frac{\partial \mathrm{f}}{\partial \boldsymbol{v}}=-J(f),
$$

for the phase-space distribution function, $f(z, v, t)$, a function of velocity $\boldsymbol{v}$ and time $t$, with spatial gradients taken along the $z$ axis, and acceleration a due to external forces, with collisional processes represented by Boltzmann's collision integral $J$. Integrating equation (1) over velocity space yields the continuity equation (here, in one dimension):

$$
\frac{\partial n}{\partial t}+\frac{\partial \Gamma}{\partial z}=S(z, t),
$$

where $n(z, t)$ is the charged-particle density, $\Gamma(z, t)=\int v_{z} f(z$, $\boldsymbol{v}, t) \mathrm{d} \boldsymbol{v}$ is the charged-particle flux in the external field direction and the right-hand side is the rate of production of particles, given by $S(z, t)=\int J_{\mathrm{NC}}(f) \mathrm{d} \boldsymbol{v}$, the integral of the non-conservative collision operator, $J_{\mathrm{NC}}$, for processes such as attachment and ionisation.

Swarm experiments are traditionally designed to operate in the hydrodynamic regime $[2,44,75]$. In this regime, the space-time dependence of $f(z, v, t)$ is a function of the number density $(n)$, and can be expressed in terms of a density gradient expansion: 


$$
\begin{aligned}
f(z, \boldsymbol{v}, t)= & n(z, t) f^{(0)}(\boldsymbol{v})-f^{(1)}(\boldsymbol{v}) \frac{\partial n(z, t)}{\partial z} \\
& +f^{(2)}(\boldsymbol{v}) \frac{\partial^{2} n(z, t)}{\partial z^{2}}+\cdots,
\end{aligned}
$$

while normalisation requires $\int f^{(j)}(\boldsymbol{v}) \mathrm{d} \boldsymbol{v}=\delta_{j 0}$. Hence, the flux and source terms in the continuity equation (2) can be identified with:

$$
\begin{aligned}
& \Gamma(z, t)=n W-D_{L} \frac{\partial n}{\partial z}+\zeta_{L} \frac{\partial^{2} n}{\partial z^{2}}-\cdots, \\
& S(z, t)=n R_{\text {net }}-S^{(1)} \frac{\partial n}{\partial z}+S^{(2)} \frac{\partial^{2} n}{\partial z^{2}}+\cdots,
\end{aligned}
$$

where $\zeta_{L}$ is the longitudinal component of the third-order transport coefficient tensor (the skewness tensor) [76]. Equation (4) is familiar as a generalisation of Fick's law. The flux drift velocity and the flux longitudinal diffusion coefficient are designated $W$ and $D_{L}$ respectively, with the net (or effective) production rate given by $R_{\text {net }}=R_{\text {ionis }}-R_{\text {att }}$. The flux transport properties in (4) and non-conservative source terms in (5) can be written in terms of the appropriate integrals of the $f^{(j)}$ appearing in equation (3), or otherwise [51, 56, 77, 78].

Substitution of equations (4) and (5) into equation (2), and grouping coefficients of gradients in the number density, yields the standard diffusion equation, when higher order terms in the hydrodynamic expansion are neglected:

$$
\frac{\partial n}{\partial t}+W_{B} \frac{\partial n}{\partial z}-D_{B, L} \frac{\partial^{2} n}{\partial z^{2}}=n R_{\text {net }},
$$

where we define the bulk $(B)$ transport coefficients in terms of the flux coefficients and the corrections due to the nonconservative source terms:

$$
\begin{gathered}
W_{B}=W+S^{(1)}, \\
D_{B, L}=D_{L}+S^{(2)} .
\end{gathered}
$$

In a drift tube experiment, $S^{(1)}$ and $S^{(2)}$ can be interpreted as modifications to the position of the centre of mass and spread about the centre of mass, respectively, arising from nonconservative processes. In general,

$$
\begin{aligned}
& S^{(1)}=\int J_{\mathrm{NC}}\left(f^{(1)}\right) \mathrm{d} \boldsymbol{v}, \\
& S^{(2)}=\int J_{\mathrm{NC}}\left(f^{(2)}\right) \mathrm{d} \boldsymbol{v} .
\end{aligned}
$$

Swarm experiments operating in the hydrodynamic regime may be analysed on the basis of the diffusion equation and hence generally sample the bulk transport coefficients. The solution of the diffusion equation for various experimental arrangements, e.g., for sources distributed in space and/or emitting for finite times, can be found by appropriate integration of this fundamental solution over space and/or time respectively, as we highlight below.
2.2. Townsend's first ionisation coefficient(s) and the fundamentally flawed Brambring equation of continuity

The Townsend ionisation coefficient is generally defined under steady state conditions. Confusion over the definition of the Townsend coefficient has however existed for an extended period, with the article by Crompton [45] representing a great overview and attempt to address this issue. In short, there are two definitions of the Townsend ionisation coefficient. The macroscopic version of Townsend's first (net) ionisation coefficient, $\alpha_{\mathrm{T}}$, (which is the difference of the ionisation and attachment (often referred to as $\eta$ ) coefficients), is defined by the relation to the particle flux (or current):

$$
\Gamma \sim \exp \left(\alpha_{\mathrm{T}} z\right)
$$

The microscopic version of the Townsend (net) ionisation coefficient, $\alpha$, is defined by the relation to the density:

$$
n(z) \sim \exp (\alpha z)
$$

The two definitions are quite different, as are their relationships to the other transport coefficients and to each other, as we explore below.

The PT experiment [58, 79-82] may be analysed using the diffusion equation (6). On the other hand, the continuity equation proposed by Brambring [1] is:

$$
\frac{\partial n}{\partial t}+\frac{\partial \Gamma}{\partial z}=\alpha_{\mathrm{T}} \Gamma
$$

that is equation (2) with a source term:

$$
S=\alpha_{\mathrm{T}} \Gamma .
$$

It is unclear from the Brambring paper [1] which form of the Townsend ionisation coefficient was proposed in their continuity equation, and perhaps their equation defines its own form of the Townsend ionisation coefficient. We do highlight, however, that the steady-state solution of equation (13) for the flux is consistent with the macroscopic form of the Townsend coefficient (11) and hence we use that form in the Brambring representation of the equation of continuity. This is a notational issue, however, which does not impact the following arguments.

Most importantly, the Brambring form of the continuity equation (13) is not derivable from the Boltzmann equation (1), except in the trivial case of no ionisation or attachment, where $S=0$. To illustrate issues associated with the Brambring representation of the equation of continuity (13), let us consider a very simple benchmark system: elastic scattering with an attachment process with a collision frequency, $\nu_{\text {att }}$, that is independent of energy. From the Boltzmann equation, the attachment collision operator has the form: $J_{\text {att }}(f)=\nu_{\text {att }} f$. The source term in the exact continuity equation (2) in the hydrodynamic regime takes the form:

$$
\begin{aligned}
S(z, t) & \equiv \int\left[J_{\text {elast }}(f)+J_{\text {att }}(f)\right] \mathrm{d} \boldsymbol{v} \\
& =0+\nu_{\text {att }} \int\left[n f^{(0)}(\boldsymbol{v})-f^{(1)}(\boldsymbol{v}) \frac{\partial n}{\partial z}\right.
\end{aligned}
$$




$$
\begin{aligned}
& \left.+f^{(2)}(\boldsymbol{v}) \frac{\partial^{2} n}{\partial z^{2}}+\cdots\right] \mathrm{d} \boldsymbol{v} \\
= & n \nu_{\text {att }} \int f^{(0)}(\boldsymbol{v}) \mathrm{d} \boldsymbol{v}-\nu_{\text {att }} \int f^{(1)}(\boldsymbol{v}) \mathrm{d} \boldsymbol{v} \frac{\partial n}{\partial z} \\
& +\nu_{\text {att }} \int f^{(2)}(\boldsymbol{v}) \mathrm{d} \boldsymbol{v} \frac{\partial^{2} n}{\partial z^{2}}+\cdots \\
= & n \nu_{\text {att }}+0+0+\cdots,
\end{aligned}
$$

where the last line follows by virtue of the normalisation condition on the $f^{(j)}$, and $J_{\text {elast }}$ denotes the elastic collision operator. In this case, $S(z, t)$ is proportional to the density with no contributions arising from the derivatives of the density. This is inconsistent with the Brambring form for the source term (14), which would have additional first and second order density spatial derivative contributions which are independent of the energy dependence of the non-conservative processes, viz substituting the expression for the flux $\Gamma$ into equation (14). Physically, if the attachment collision frequency is independent of energy then it cannot modify the position of the centre of mass (first moment of the density) nor the rate of spread/diffusion (second moment of the density) of the pulse. In contrast, the Brambring equation has explicit modifications to both of these moments of the pulse, whenever there are non-conservative processes operative, irrespective of the energy dependence of the non-conservative collision frequency.

While the Brambring representation of the equation of continuity is thus fundamentally flawed from a physical viewpoint, in the following sections, we highlight how to relate the transport properties extracted from the PT experiment via an analysis using the Brambring representation of the continuity equation (and related equation for the measurable current in the external circuit), with the standard definition of the transport coefficients from a Boltzmann equation/kinetic theory perspective. This will have importance for the application of PT transport properties in fluid/moment models of plasmas for example, as well as for the extraction of cross-sections through the swarm inversion process.

\section{Interpreting transport coefficients from the measured current in the pulsed-Townsend experiment}

\subsection{Solution of the standard diffusion equation}

Firstly, consider an idealised TOF experiment in a finite geometry $0 \leqslant z \leqslant L$, in which a sharp pulse of $n_{0}$ charge carriers is released from a source plane $z=z_{0}$ at time $t=t_{0}$, i.e.,

$$
n\left(z, t_{0}\right)=n_{0} \delta\left(z-z_{0}\right)
$$

The solution of the diffusion equation (6) for $z_{0}=0, t_{0}=0$ and infinite geometry $(L \rightarrow \infty)$ is the well known travelling pulse $[2,53]$ :

$$
n(z, t)=\frac{n_{0} \exp \left(R_{\mathrm{net}} t\right)}{\sqrt{4 \pi D_{B, L} t}} \exp \left[-\frac{\left(z-W_{B} t\right)^{2}}{4 D_{B, L} t}\right]
$$

In finite geometry, assuming perfectly absorbing boundaries, and

$$
n(0, t)=0=n(L, t),
$$

the solution of the diffusion equation may be obtained using the Poisson summation theorem [73, 74] as,

$$
\begin{aligned}
n\left(z, t ; z_{0}, t_{0}\right)= & \frac{n_{0}}{\sqrt{4 \pi D_{B, L}\left(t-t_{0}\right)}} \exp \\
& \times\left\{R_{\mathrm{net}} t+\frac{W_{B}}{2 D_{B, L}}\left[z-z_{0}-\frac{1}{2} W_{B}\left(t-t_{0}\right)\right]\right\} \\
& \times \sum_{j=-\infty}^{\infty}\left\{\exp \left[-\frac{\left(z-z_{0}-2 j L\right)^{2}}{4 D_{B, L}\left(t-t_{0}\right)}\right]\right. \\
& \left.-\exp \left[-\frac{\left(z+z_{0}-2 j L\right)^{2}}{4 D_{B, L}\left(t-t_{0}\right)}\right]\right\} .
\end{aligned}
$$

One can consider more elaborate boundary conditions, however for the current study the simplified boundary conditions (18) are sufficient.

It is convenient for the purposes of the following discussion to consider the situation where the left hand boundary recedes to $-\infty$. This may be achieved mathematically by an appropriate transformation of coordinates, in which $L$ now denotes the distance of the right hand boundary from the source, which is now located at the origin of coordinates. Equation (19) then becomes, with $t_{0}=0$,

$$
\begin{aligned}
n(z, t ; L)= & \frac{n_{0} \exp \left(R_{\mathrm{net}} t+\frac{W_{B}}{2 D_{B, L}}\left(z-\frac{1}{2} W_{B} t\right)\right)}{\sqrt{4 \pi D_{B, L} t}} \\
& \times\left\{\exp \left[-\frac{z^{2}}{4 D_{B, L} t}\right]-\exp \left[-\frac{(z-2 L)^{2}}{4 D_{B, L} t}\right]\right\},
\end{aligned}
$$

describing the spatio-temporal variation of $n(z, t)$ in a TOF drift tube.

\subsection{Extracting bulk transport coefficients from the pulsed-Townsend experiment}

Consider now the PT experiment - a plane parallel swarm system where all spatial variation is confined to the $z$ direction, normal to the electrodes. Under typical measurement conditions, the transit time of the electrons is much less than the $R C$ time constant of the circuit [2] and the current in the external circuit is given by:

$$
I=\frac{q}{L} \int_{0}^{L} \Gamma\left(z^{\prime}, t\right) \mathrm{d} z^{\prime} .
$$

Using Fick's law (4), this can be written in terms of the transport coefficients and is given by:

$$
I(t)=\frac{q W}{L} \int_{0}^{L} n(z, t) \mathrm{d} z
$$

where the diffusive contribution has been eliminated due to the relation: 


$$
\int_{0}^{L} D_{L} \frac{\partial n}{\partial z} \mathrm{~d} z=D_{L} n(L)-D_{L} n(0)=0,
$$

for perfectly absorbing boundary conditions. It follows from (22) that the measurable current in the external circuit is given by:

$$
\begin{aligned}
I(t)= & \frac{n_{0} q W}{2 L} \exp \left(R_{\mathrm{net}} t\right)\left\{\left[1-\phi\left(\frac{W_{B} t-L}{\sqrt{4 D_{B, L} t}}\right)\right]\right. \\
& \left.+\exp \left(\left[\frac{W_{B}}{D_{B, L}}\right] L\right)\left[\phi\left(\frac{W_{B} t+L}{\sqrt{4 D_{B, L} t}}\right)-1\right]\right\},
\end{aligned}
$$

where $\phi$ represents the error function. Hence, full current transients for the current in the external circuit of the PT experiment fitted to equation (24) can yield the bulk transport coefficients: the net ionisation rate coefficient $R_{\text {net }}=R_{\text {ionis }}-R_{\text {att }}$, the bulk drift velocity $W_{B}$, and the bulk diffusion coefficient $D_{B, L}$. Even though the current scales with the flux drift velocity, $W$, the time-dependence of the measured current is determined by the bulk transport coefficients $-R_{\text {net }}, W_{B}$ and $D_{B, L}$ via (24). This is consistent with the conclusions of Blevin and Fletcher [50] and Robson [53]. If the initial number of electrons is known, then we can also simultaneously extract the flux drift velocity from an analysis of the current in the external circuit. This provides an additional transport coefficient that can be used for cross-section fitting/extraction from swarm experiments.

\section{Relating existing PT transport properties to the standard transport coefficient definitions}

Given the wealth of experimental work and associated extraction of transport properties and fitting of cross-sections to the PT data, the obvious question remaining is how do we relate the PT experimental transport properties to the transport coefficients which are grounded in the Boltzmann equation/kinetic theory.

Here, we return to the Brambring representation of the equation of continuity (13) and find the equivalent expression for the current in an external circuit ${ }^{12}$. If we substitute Fick's law expression (4) into the Brambring equation (13) (retaining

\footnotetext{
12 While the functional form of the current in the external circuit and its relation to the transport coefficients can be a source of uncertainty, these are distinct from the uncertainties that can typically be obtained from approximate analysis of the current in the external circuit. Indeed, some analyses have used quite simplified approaches to extract the various transport properties from the current in the external circuit [58, 65, 79, 83-85], which may lead to further issues.

For example, extracting $\tilde{W}$, through dividing $L$ by the measured electron transit time, $T_{\mathrm{e}}$, where the transit time is defined as the difference in times between the measured current's rise and fall to the respective half values. This is a measure of a drift velocity, but not one that is consistent with the flux or bulk drift velocities, or equation (24). Using that $\tilde{W}$ to then determine any further parameters (e.g. $\tilde{\alpha}_{\mathrm{T}}$ or $\tilde{D}_{L}$ ) will further propagate uncertainties in the other derived coefficients/parameters. Non-linear curve fitting to the full equation (24) should in fact be performed (as in, for example, reference [81]) in all cases.
}

only first order terms in the density gradient expansion ${ }^{13}$ ), on re-arranging we obtain the diffusion-type equation:

$$
\frac{\partial n}{\partial t}+\left[\tilde{W}+\tilde{\alpha}_{\mathrm{T}} \tilde{D}_{L}\right] \frac{\partial n}{\partial z}-\tilde{D}_{L} \frac{\partial^{2} n}{\partial z^{2}}=n \tilde{\alpha}_{\mathrm{T}} \tilde{W} .
$$

The tildes here denote transport properties arising from the Brambring representation of the diffusion equation. Since the Brambring representation of the equation of continuity (13) is not derivable from Boltzmann's equation/kinetic theory, the terms drift velocity, diffusion coefficient and alpha as defined by the Brambring representation do not have a standard kinetic theory definition (i.e. are not representable in terms of an integral of the phase-space distribution function and hence cannot be found directly in terms of a solution of Boltzmann's equation or Monte Carlo simulation) when non-conservative processes are operative and hence may not have the standard meaning of drift velocity, diffusion, etc, under such conditions.

Following the same procedure as above, but using the Brambring representation of the diffusion equation (25) instead of the conventional diffusion equation (6), it follows that the functional form of the current in the external circuit is given by:

$$
\begin{aligned}
I(t)= & \frac{n_{0} q W}{2 L} \exp \left(\tilde{\alpha}_{\mathrm{T}} \tilde{W} t\right) \\
& \times\left\{\left[1-\phi\left(\frac{\left(\tilde{W}+\tilde{\alpha}_{\mathrm{T}} \tilde{D}_{L}\right) t-L}{\sqrt{4 \tilde{D}_{L} t}}\right)\right]\right. \\
& +\exp \left(\left[\frac{\tilde{W}+\tilde{\alpha}_{\mathrm{T}} \tilde{D}_{L}}{\tilde{D}_{L}}\right] L\right) \\
& \left.\times\left[\phi\left(\frac{\left(\tilde{W}+\tilde{\alpha}_{\mathrm{T}} \tilde{D}_{L}\right) t+L}{\sqrt{4 \tilde{D}_{L} t}}\right)-1\right]\right\} .
\end{aligned}
$$

This is the same expression as that from the original Brambring paper (see equation (12) of reference [1]) and used by the experimental groups $[58,79,83,84]$, expressed using the PT transport properties. It is important to note that the $W$ appearing in the first factor on the rhs of equation (26) is the flux drift velocity $W$, not the bulk drift velocity $W_{B}$ or the PT transport property $\tilde{W}$.

The key to relating the PT transport properties to the standard transport coefficients is to understand how they are extracted from the fitting of the current in the external circuit in a typical analysis of the PT experiment. The expression for the current in the external circuit, whether it be the expression

In addition, extracting $\tilde{\alpha}_{\mathrm{T}}$ from the rising component of the measured current [58] (and $\tilde{W}$ from the earlier step) fails to capture the diffusion contributions to the current in the external circuit.

Using these simplified processes to establish initial estimates of the parameters, to start the non-linear curve fitting of the measured current (as in reference [81], for example) is, however, good practice.

${ }^{13}$ This representation of the current in the external circuit fails to capture the second order contributions to the source term and hence the equation cannot be an accurate representation of the experimentally measured current in the external circuit when the product $\alpha_{\mathrm{T}} \zeta_{L}$ becomes appreciable relative to $D_{L}$. While measurement of the skewness term has not been performed to date, many transient and stationary effects may skew the profile and require consideration [76, $86,87]$. 
arising from the diffusion equation (24) or the expression arising from the unphysical Brambring equation (26), takes the same general form, i.e. is mathematically equivalent:

$$
\begin{aligned}
I(t)= & a \exp (b t)\left\{\left[1-\phi\left(\frac{c t-L}{\sqrt{4 \mathrm{~d} t}}\right)\right]\right. \\
& \left.+\exp \left(\left[\frac{c}{d}\right] L\right)\left[\phi\left(\frac{c t+L}{\sqrt{4 \mathrm{~d} t}}\right)-1\right]\right\},
\end{aligned}
$$

where $a, b, c$ and $d$ can be found from the non-linear curve fitting procedure and are related to the standard bulk transport coefficients/PT transport properties through comparisons with equations $(24) /(26)$, or equivalently through comparison of equations $(6) /(25)$. If in the previous analyses of PT experiments expression (26) has been fitted to the current in the external circuit, it then follows that the PT transport properties $\left(\tilde{\alpha}_{\mathrm{T}}, \tilde{W}, \tilde{D}_{L}\right)$ can be related to the bulk transport coefficients $\left(R_{\text {net }}, W_{B}, D_{B, L}\right)$ via $^{14}$ :

$$
\begin{aligned}
R_{\mathrm{net}} & =\tilde{\alpha}_{\mathrm{T}} \tilde{W}=b, \\
W_{B} & =\tilde{W}+\tilde{\alpha}_{\mathrm{T}} \tilde{D}_{L}=c, \\
D_{B, L} & =\tilde{D}_{L}=d .
\end{aligned}
$$

If the initial number of electrons $n_{0}$ is measured accurately, the fitting parameter $a$ can provide a technique to measure the flux drift velocity, $W$ - the first experiment able to do so!

We now consider some examples, transforming the PT transport properties extracted from existing PT measurements, through implementation of the theoretical relationships (30)-(32), in order to compare with the bulk transport coefficients which have firm foundations in kinetic theory/Boltzmann's equation. TOF measurements are included as measurements of bulk coefficients since they are analysed according the diffusion equation (6). It is important to note:

- The relationships (e.g. $W_{B}=\tilde{W}+\tilde{\alpha}_{\mathrm{T}} \tilde{D}_{L}$ ) are presented only when all transport properties from the PT experiment $\left(\tilde{W}, \tilde{\alpha}_{\mathrm{T}}, \tilde{D}_{L}\right)$ are available from a single study to do the transformation

- The intent of this section is purely to highlight the validity of the relationship between the PT transport properties and the bulk transport coefficients. Hence,

- We do not preference any particular measurement technique over the other, but rather focus on the presentation of coefficients with firm theoretical foundations. Assessment of the quality of any experimental measurements is beyond the scope of the present work.

\footnotetext{
${ }^{14}$ It is important to make the distinction that the relationship (30), $R_{\text {net }}=\tilde{\alpha}_{\mathrm{T}} \tilde{W}$, is valid for the PT transport properties $\tilde{\alpha}_{\mathrm{T}}$ and $\tilde{W}$. The relationship between $R_{\text {net }}$, the SST $\alpha_{\mathrm{T}}$ and the bulk transport coefficients, however, is given by $[53,56]$.

$$
R_{\text {net }}=\alpha_{\mathrm{T}} W_{B}-\alpha_{\mathrm{T}}^{2} D_{B, L}+\cdots
$$

which, in the limit of small diffusion, may be approximated by

$$
R_{\mathrm{net}} \approx \alpha_{\mathrm{T}} W_{B}
$$

- Transport coefficient calculations are obviously dependent on the cross-section set used. The calculations presented here are for comparison with the transformed PT results and should not be interpreted as the reference for quality of the experimental results or analysis.

- The error propagation associated with the application of the theoretical relationships (30)-(32) on existing PT transport properties results in large error bars. Ideally, reanalysis of the PT experiment current transients according to equation (24) would be preferred, if available, for appropriate determination of experimental error.

In the following subsections we present the results for Ar and $\mathrm{SF}_{6}$. Ar is considered somewhat of a benchmark gas known for high accuracy measurements and well known crosssections [33], while $\mathrm{SF}_{6}$ provides a good example of when the differences are quite important due to its strong electron attachment and ionisation.

The numerical methods employed in the solution of Boltzmann's equation (1) for the calculated coefficients have been described in detail previously, and the reader is referred to references $[88,89]$.

\subsection{Argon}

Figures 1 and 2 present some of the available experimentally measured bulk drift velocities, $W_{B}$, and ionisation rate coefficients, $R_{\text {ionis }}$, for electron swarms in Ar. In the upper panel of figure 1, the $W_{B}$ transformed via the theoretical relationship (31) from the $\tilde{W}$ extracted from the PT experiment of de Urquijo et al [90] and Hernández-Ávila et al [91, 92] are shown with the $W_{B}$ measured from the TOF apparatus of Kücükarpaci and Lucas [93, 94] and Nakamura and Kurachi [37], and the $W_{B}$ from the scanning drift tube measurements of Korolov et al [69]. Measurements of $W_{B}$ are sparse in the $E / n_{0}$ region where the transformation is most pronounced, although the trend of the de Urquijo et al $W_{B}$ lies somewhat above the highest $W_{B}$ datum of Nakamura and Kurachi [37] at $50 \mathrm{Td}$, the Kücükarpaci and Lucas $[93,94] W_{B}$ at around $150 \mathrm{Td}$, and the measurements of Korolov et al [69]. In contrast, both the $\tilde{W}$ and $W_{B}$ measurements of Hernández-Ávila et al [91, 92] tend to lie below the 50 Td Nakamura and Kurachi [37] value and the measurements of Korolov et al [69], with the highest $E / n_{0}$ measurement of Kücükarpaci and Lucas $[93,94]$ in good agreement with the transformed $W_{B}$. For Ar, the $\tilde{W}$ and $W_{B}$ differ by up to $4.2 \%$ for the de Urquijo et al [90] measurements and up to $8.2 \%$ for the Hernández-Ávila et al [91, 92] measurements, due to the relative magnitudes of the $D_{B, L}$ and $\tilde{\alpha}$ (as shown in figure 11 of de Urquijo et al [90]), the difference increasing with $E / n_{0}$. These results highlight the accuracy of the PT measurements and associated analysis in reference [90].

The bulk and flux drift velocity calculated using the crosssection set extracted from Magboltz [95] are also displayed in figure 1 for comparison. While these calculations are dependent on the cross-section set utilised, good agreement is observed between the calculated $W_{B}$ and the $W_{B}$ transformed from the PT measurements of de Urquijo et al [90]. 

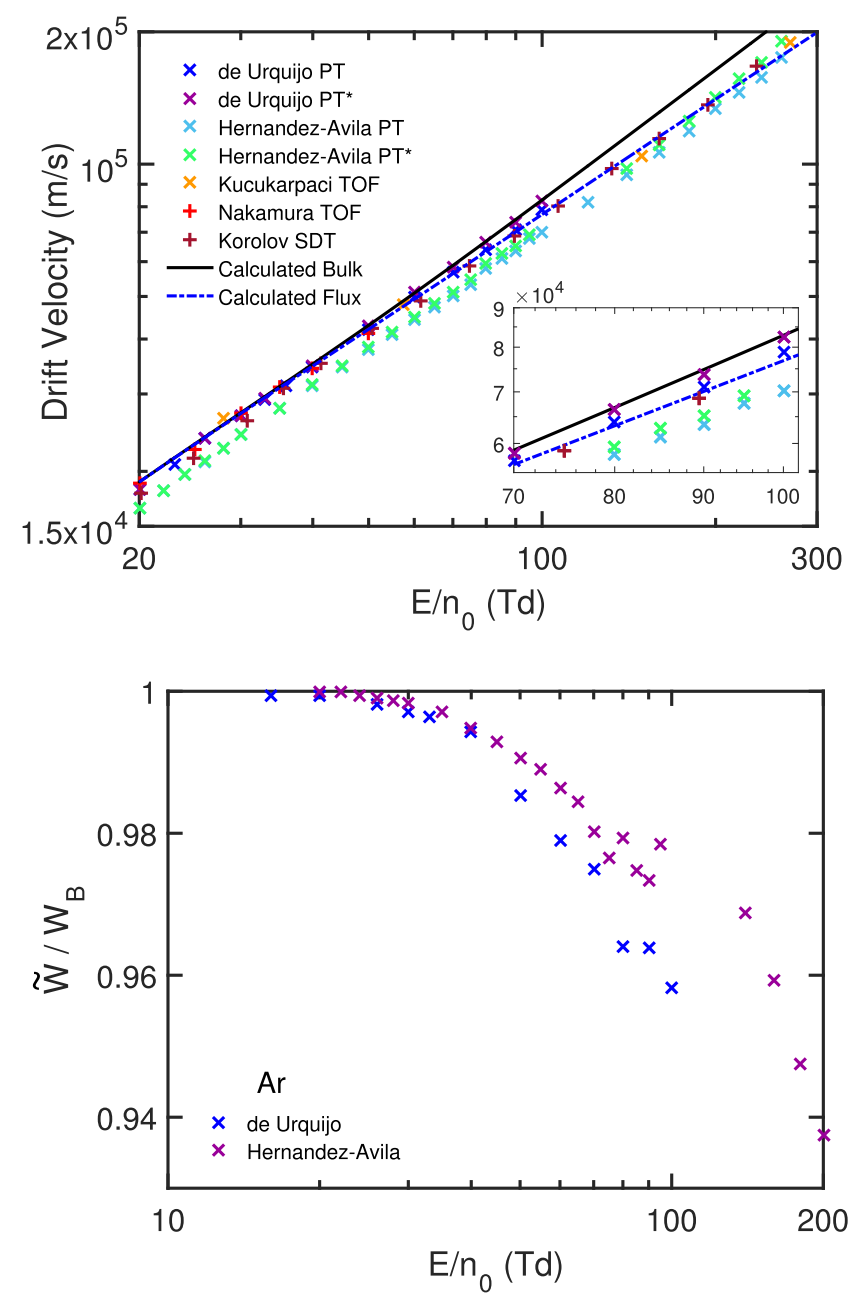

Figure 1. The drift velocity for electron swarms in gaseous argon. For the PT measurements, the bulk drift velocity $W_{B}$ has been transformed from the PT measurements of $\tilde{W}$ through the theoretical relationship (31), and is denoted by the asterisk $\left(^{*}\right)$. (Upper) The $W_{B}$ transformed from the PT measurements of de Urquijo et al [90] (transformed using the $D_{B, L}$ first reported in reference [97] although recorded with the $\tilde{W}$ and $\tilde{\alpha}_{\mathrm{T}}$ reported in reference [90]) and Hernández-Ávila et al $[91,92]$ are compared with the $W_{B}$ TOF measurements of Kücükarpaci and Lucas $[93,94]$ and Nakamura and Kurachi [37], and the $W_{B}$ from the scanning drift tube (SDT) apparatus of Korolov et al [69]. (Lower) The ratio of the PT-measured to bulk drift velocities, $\tilde{W} / W_{B}$, for the measurements of de Urquijo et al [90] and Hernández-Ávila et al [91, 92].

'Calculated' represents the flux and bulk drift velocities calculated from a solution of the Boltzmann equation using the cross-sections extracted from Magboltz [95].

The lower panel of figure 1 displays the ratio of the PT drift measurement to the bulk drift velocity, $\tilde{W} / W_{B}$. The ratio illustrates the difference between the drift velocities, which increases with increasing $E / n_{0}$, as expected from the increasing magnitude of the $\tilde{\alpha}_{\mathrm{T}} \tilde{D}_{L}$ term.

Figure 2 shows a comparison of $R_{\text {ionis }}$ in Ar which is, to our knowledge, limited to the transformed PT measurements of de Urquijo et al [90] and Hernández-Ávila et al [91, 92], through the theoretical relationship (30), and the $R_{\text {ionis }}$ extracted directly from the PT measurements of Dahl et al [58] and Haefliger and Franck $[63,96]$. At the lower $E / n_{0}$ of the

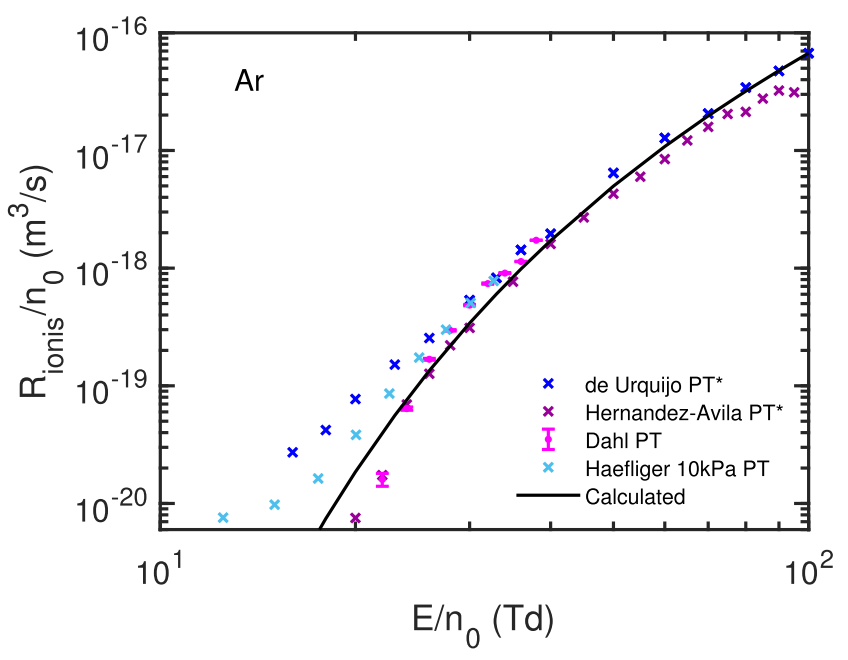

Figure 2. The ionisation rate coefficient for electron swarms in gaseous argon. The $R_{\text {ionis }}$ transformed from the PT measurements of de Urquijo et al [90] and Hernández-Ávila et al [91, 92], through the theoretical relationship (30) and denoted by the asterisk $\left({ }^{*}\right)$, are compared with the (positive) $R_{\text {ionis }}$ values reported in Dahl et al [58] and Haefliger and Franck $[63,96]$ (the representative measurement at $10 \mathrm{kPa}$ has been used). 'Calculated' represents the $R_{\text {ionis }}$ calculated from a solution of the Boltzmann equation using the cross-sections extracted from Magboltz [95].

de Urquijo et al measurements, good agreement is observed with the majority of the Haefliger and Franck coefficients (measured over a range of pressures, although only the $10 \mathrm{kPa}$ measurement is displayed in figure 2), and over intermediate $E / n_{0}$ with the Dahl et al measurements. Over the full $E / n_{0}$ range of the (positive) Dahl et al measurements, very good consistency with the transformed Hernández-Ávila et al $[91,92] R_{\text {net }}$ is observed.

\section{2. $\mathrm{SF}_{6}$}

Figures 3 and 4 present the bulk drift velocity, $W_{B}$, and net rate coefficient, $R_{\text {net }}$, from some of the available PT and TOF measurements for electron swarms in $\mathrm{SF}_{6}$. The upper panel of figure 3 includes $W_{B}$ transformed from the $\tilde{W}$ measurements of Aschwanden [66] and Xiao et al [98], via the theoretical relationship (31), and the $W_{B}$ TOF measurements of Nakamura [99] and Naidu and Prasad [100]. The transformation to $W_{B}$ from the measured $\tilde{W}$ of both Aschwanden and Xiao et al results in a decrease in magnitude below $361 \mathrm{Td}$, a consequence of the attachment-dominated $\tilde{\alpha}$, and increase in magnitude above this $E / n_{0}$ as ionising collisions dominate the $\tilde{\alpha}$ (and similarly, $R_{\text {net }}$ in the lower panel of figure 3 ). The transformation of the Aschwanden [66] drift velocity results in a decrease of up to $4.7 \%$ in the attachment-dominated region, and an increase of up to $9.2 \%$ in the ionisation-dominated region, at the highest $E / n_{0}$, while the transformation of the Xiao et al [98] measurements results in a decrease of up to $5.2 \%$ in the attachment-dominated region, and an increase of up to $2.3 \%$ in the ionisation-dominated region. The results shown in the upper panel of figure 3 highlight, in particular, the accuracy of the PT measurements and associated analysis in the work of Aschwanden [66]. 

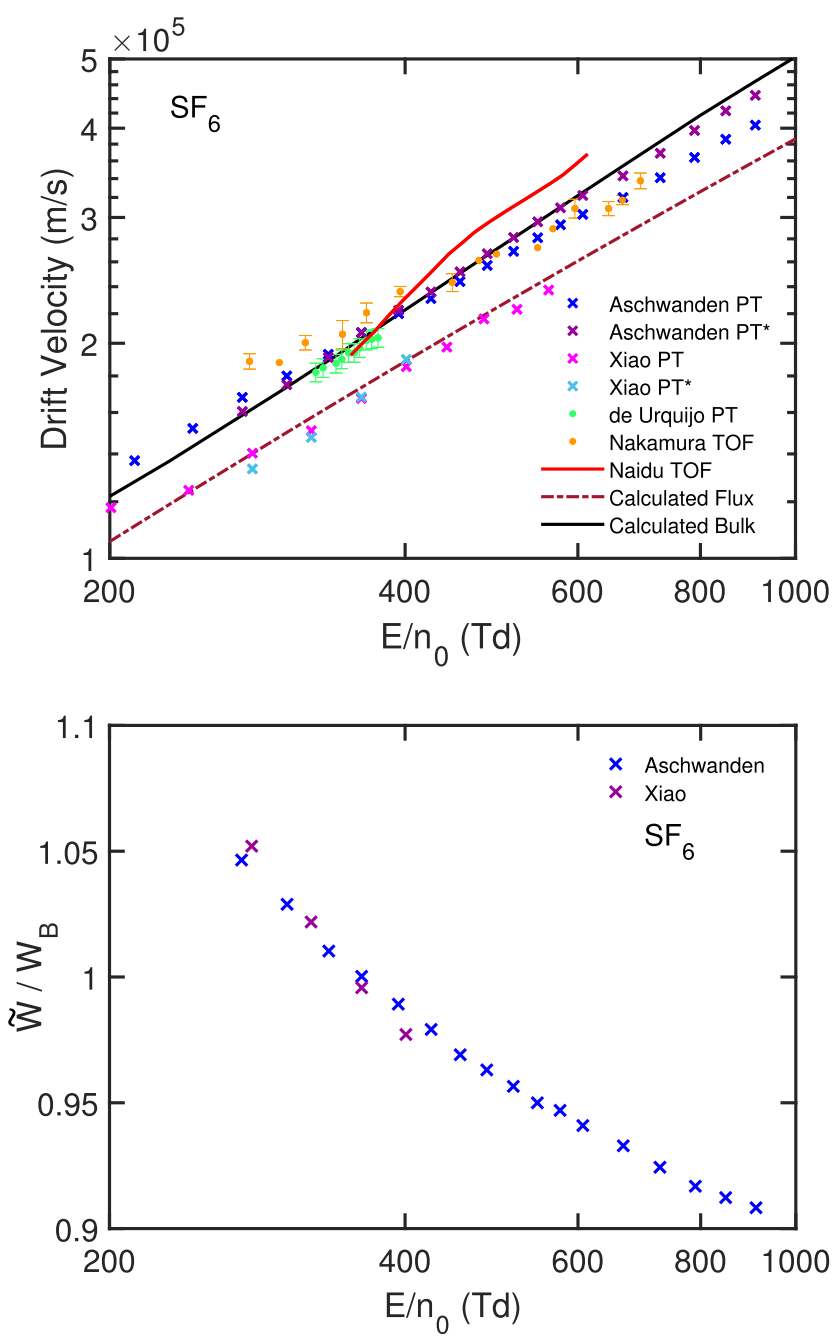

Figure 3. The drift velocity for electron swarms in gaseous $\mathrm{SF}_{6}$. For the PT measurements, the bulk drift velocity $W_{B}$ has been transformed from the PT measurements of $\tilde{W}$ through the theoretical relationship (31), and is denoted by the asterisk $\left(^{*}\right)$. (Upper) The $W_{B}$ transformed from the PT measurements of Aschwanden [66] (with the necessary PT transport properties available for $E / n_{0} \geqslant 273 \mathrm{Td}$ ) and Xiao et al [98] (with the necessary properties available for $E / n_{0}=279-401 \mathrm{Td}$ ), are shown alongside the $\tilde{W}$ measured by the PT apparatus of de Urquijo et al [103] (where, in the absence of $D_{B, L}, W_{B}$ could not be determined). Also displayed are the $W_{B}$ TOF measurements of Nakamura [99] and Naidu and Prasad [100] (digitised from Christophorou and Olthoff [102]). (Lower) The ratio of the PT-measured to bulk drift velocities, $\tilde{W} / W_{B}$, for the PT measurements of de Urquijo et al [103], Xiao et al [98] and Xiao et al [104]. 'Calculated' represents the flux and bulk drift velocities calculated from a solution of the Boltzmann equation using the cross-section data of Biagi [101] from the LXCat database.

Compared to the other $W_{B}$ measurements (from the TOF apparatus), the transformation of the Aschwanden data increases the differences when compared to the measurements of Nakamura over all $E / n_{0}$, but decreases the differences from the Naidu and Prasad $W_{B}$. Similarly, for the four data points of the Xiao et al measurements, where all PT transport properties were reported (279-401 Td), the transformation to $W_{B}$ increases the differences from the other experimental measurements.

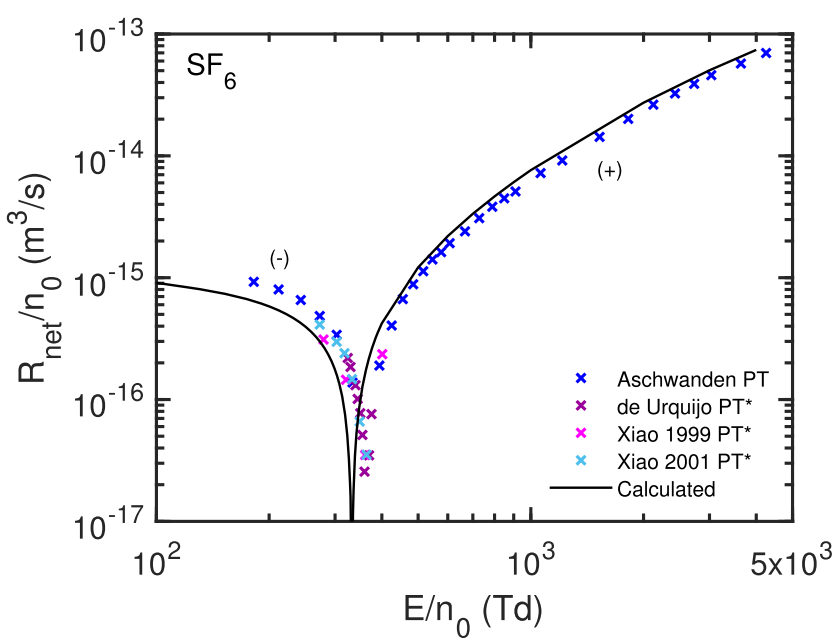

Figure 4. The ionisation coefficient for electron swarms in gaseous $\mathrm{SF}_{6}$. The absolute value of the $R_{\text {net }}$ reported from the PT measurements of Aschwanden [66] are compared with the $R_{\text {net }}$ transformed through the theoretical relationship (30) and denoted by the asterisk $\left(^{*}\right)$, from the PT measurements of de Urquijo et al [103], Xiao et al [98] and Xiao et al [104]. 'Calculated' represents the $R_{\text {net }}$ calculated from a solution of the Boltzmann equation using the cross-section data of Biagi [101] from the LXCat database.

We are thus unable to reconcile the PT measurements of $\tilde{W}$ from Xiao et al.

The flux and bulk drift velocity and $R_{\text {net }}$ values calculated using the cross-section data of Biagi [101] from the LXCat database, are also shown in figures 3 and 4. These representative calculations are dependent on the cross-section set used, and are included only to indicate the magnitudes of the transport coefficients, in particular highlighting the effect of the non-conservative processes on the calculated $W_{B}$ compared to the flux drift velocity, $W^{15}$. In both the attachment and ionisation-dominated regions, the transformation of the Aschwanden $\tilde{W}$ changes in the direction consistent with the representative calculations. As a result, the transformed experimental results of Aschwanden are in good agreement with our representative calculations.

The lower panel of figure 3 displays the ratio of the PT drift measurement to the bulk drift velocity, $\tilde{W} / W_{B}$. The ratio illustrates the contribution of the $\tilde{\alpha}_{\mathrm{T}} \tilde{D}_{L}$ term in relationship (30), to the transformation to $W_{B}$. The change in sign of $R_{\text {net }}$ results in a decrease to $W_{B}$ relative to the measured $\tilde{W}$ for the lower $E / n_{0}$, followed by an increase of $W_{B}$ relative to $\tilde{W}$. The magnitude of the difference increases further from the breakdown $E / n_{0}$ value, consistent with the magnitude of $R_{\text {net }}$.

In the absence of any other $R_{\text {net }}$ measurements in $\mathrm{SF}_{6}$, to our knowledge ${ }^{16}$, figure 4 only displays the rate coefficient of Aschwanden [66], reported directly in that thesis,

${ }^{15}$ We make no comment on the validity of that cross-section set here, only to highlight that we are unable to predict accurately the breakdown reduced field as shown in figure 4.

${ }^{16}$ We note that many values of the attachment rate coefficient have been reported for $\mathrm{SF}_{6}$ in various buffer gases (see the review of Christophorou and Olthoff [102]), but in the absence of any mean energy values for the PT measurements, the comparison of $R_{\text {att }}$ at a common mean energy cannot be made. 
and the rate coefficients of de Urquijo et al [103] and Xiao et al [98, 104], transformed through the relationship (30), with good agreement observed between all.

\section{Concluding remarks}

In this study we have addressed, from a fundamental viewpoint, the issue of the analysis and interpretation of the PT experiment. We have shown that the governing equation traditionally used to analyse the PT experiment — the Brambring representation of the equation of continuity - is fundamentally flawed, and transport properties subsequently defined through that equation do not have a clear representation in terms of the distribution function. We have presented an expression for the current in the external circuit of the PT experiment in terms of the standard diffusion equation and the universal transport coefficients defined through it-the bulk transport coefficients. In addition, we have developed a relationship between the transport properties extracted from the PT experiment using the Brambring representation of the equation of continuity and the bulk transport coefficients, and highlighted the validity of the relationship for various gases. Given the errors that are necessarily propagated through this process, we suggest that all previous transport properties extracted from PT experimental data where non-conservative processes are operative be re-analysed according to the diffusion equation based current expression to enable measurement of standard bulk transport coefficients prior to any subsequent application (e.g. evaluating complete and accurate sets of scattering crosssections, and further utilisation in modelling of plasmas and ionised gases [3, 12, 13, 44, 105]).

A consequence of the present analysis is the necessary reconciliation between experimental and theoretical studies involving PT measurements.

As a minimum for any swarm study, the exact definition of any transport property/coefficient measured or used for further analyses must be identified. Further, the definition of any transport property/coefficient must be consistent with those defined through kinetic theory and representable in terms of the phase-space distribution function.

In addition, the following are recommended for clear identification in experimental studies:

- Primary reference to the exact source equation used for analysis, and any assumptions entailed.

- A description of the method of the analysis of all measurements.

- A detailed estimate of the error associated with the statistical analysis (including systematic errors, reproducibility, etc), alongside the reported experimental uncertainty.

- The applicability of the hydrodynamic or nonhydrodynamic regime, and the methods used to ensure sampling under appropriate conditions. This point is generally well accomplished in the literature.

- Clear uncertainty estimates of all elements (e.g. pressure, mixture ratio, etc) and how they propagate through to the final result [106-109].
Prior to the use of any swarm transport measurements in theoretical models (low temperature plasma models), the effect of non-conservative collisions must be identified, since it is when flux and bulk values start to differ significantly that one needs to pay attention to the nature of the transport data required in their models. A detailed prescription has been presented previously [110].

Using 'wrong' theory yields results that may be up to a factor of 10 different under some circumstances, though often effects are of the order of $10 \%-30 \%$. However, if one uses a similar theory to implement the cross-sections obtained from incorrectly interpreted data one returns to the original experimental data. Plasma modelling is sufficiently robust that small changes in the transport data are easily compensated by small self consistent adjustments of the local field. Problems occur when one uses more exact models to describe plasmas. PIC codes with a properly implemented and tested Monte Carlo simulation will provide correct calculation of fluxes and thus the effect of the cross-sections obtained from the incorrectly interpreted data may become large, as stated above. Even more so, as the plasma field is calculated self consistently small changes in the local $E / n_{0}$ as compared to the properly determined values would originate. Some processes with a high threshold, such as dissociation and ionization, are very strongly affected by the local normalised electric field, even by orders of magnitude (see reference [111], for example).

In this vein, it is recommended that theoretical studies clearly identify the definition of any utilised experimental measurements and any further analysis of those measurements (e.g. transformation from one transport coefficient to another via approximate relationships with associated errors propagated).

In addition to these recommendations to aid reconciliation within the literature, the present work also seeds further investigation, specifically into the analysis and interpretation of the PT experiment. For example, the impact of the boundary conditions, on the electron density at the electrodes, on the expression for the current in the external circuit, and the ability to extract higher order transport coefficients (e.g. skewness) from the current measured from the PT experiment should also be studied.

\section{Acknowledgments}

The authors would like to thank the Australian Research Council through its Discovery Programme (DP180101655) for financial support. S Dujko, D Bošnjaković and I Simonović are supported by the Ministry of Education, Science and Technological Development of the Republic of Serbia and the Institute of Physics (Belgrade), while Z Lj Petrović is grateful to the SASA F155 project and Ulster University for support.

\section{Data availability statement}

The data that support the findings of this study are available upon reasonable request from the authors. 


\section{ORCID iDs}

M J E Casey (iD https://orcid.org/0000-0003-0193-211X

P W Stokes (iD https://orcid.org/0000-0002-0956-5927

D G Cocks (D) https://orcid.org/0000-0002-9943-7100

D Bošnjaković (D) https://orcid.org/0000-0002-2725-5287

I Simonović (D) https://orcid.org/0000-0001-6704-9042

M J Brunger (D) https://orcid.org/0000-0002-7743-2990

S Dujko (D) https://orcid.org/0000-0002-4544-9106

Z Lj Petrović (D) https://orcid.org/0000-0001-6569-9447

R E Robson (ID https://orcid.org/0000-0003-2613-4229

R D White (D) https://orcid.org/0000-0001-5353-7440

\section{References}

[1] Brambring J R 1964 Z. Phys. 179532

[2] Huxley L G H and Crompton R W 1974 The Diffusion and Drift of Electrons in Gases (New York: Wiley)

[3] Adamovich I et al 2017 J. Phys. D: Appl. Phys. 50323001

[4] Brunger M J 2017 Int. Rev. Phys. Chem. 36333

[5] Campbell L and Brunger M J 2013 Plasma Sources Sci. Technol. 22013002

[6] Brunetti R et al 2005 New Astron. Rev. 49265

[7] Calvo J et al 2017 J. Cosmol. Astropart. Phys. JCAP03(2017) 003

[8] Montie T C, Kelly-Wintenberg K and Roth J R 2000 IEEE Trans. Plasma Sci. 2841

[9] Bekeschus S, Schmidt A, Weltmann K-D and von Woedtke T 2016 Clin. Plasma Med. 419

[10] von Woedtke T, Metelmann H-R and Weltmann K-D 2014 Contrib. Plasma Phys. 54104

[11] Stoffels E, Sakiyama Y and Graves D B 2008 IEEE Trans. Plasma Sci. 361441

[12] Muñoz A, Blanco F, Garcia G, Thorn P A, Brunger M J, Sullivan J P and Buckman S J 2008 Int. J. Mass Spectrom. 277 175

[13] Francis Z, Incerti S, Capra R, Mascialino B, Montarou G, Stepan V and Villagrasa C 2011 Appl. Radiat. Isot. 69220

[14] Rabie M and Franck C M 2018 Environ. Sci. Technol. 52369

[15] Bartschat K and Kushner M J 2016 Proc. Natl Acad. Sci. USA 1137026

[16] Kong M G, Kroesen G, Morfill G, Nosenko T, Shimizu T, van Dijk J and Zimmermann J L 2009 New J. Phys. 11115012

[17] Christophorou L G and Olthoff J K 2001 Advances in Atomic, Molecular, and Optical Physics vol 44 (Amsterdam: Elsevier) pp 59-98

[18] Brandenburg R 2017 Plasma Sources Sci. Technol. 26053001

[19] Roussel-Dupré R, Colman J J, Symbalisty E, Sentman D and Pasko V P 2008 Space Sci. Rev. 13751

[20] Misra N N, Martynenko A, Chemat F, Paniwnyk L, Barba F J and Jambrak A R 2018 Crit. Rev. Food Sci. Nutr. 581832

[21] Marinković B P, Bredehöft J H, Vujčić V, Jevremović D and Mason N J 2017 Atoms 546

[22] Frost L S and Phelps A V 1962 Phys. Rev. 1271621

[23] Engelhardt A G and Phelps A V 1964 Phys. Rev. 133 A375

[24] Engelhardt A G, Phelps A V and Risk C G 1964 Phys. Rev. 135 A1566

[25] Phelps A V 1968 Rev. Mod. Phys. 40399

[26] Christophorou L G and Hunter S R 1984 Electron Molecule Interactions and Their Applications vol 2 ed L G Christophorou (New York: Academic Press)

[27] Milloy H B and Crompton R W 1977 Phys. Rev. A 151847

[28] Crompton R W 1994 Advances in Atomic, Molecular, and Optical Physics vol 32 (Amsterdam: Elsevier) pp 97-148
[29] Petrović Z Lj, Šuvakov M, Nikitović Ž, Dujko S, Šašić O, Jovanović J, Malović G and Stojanović V 2007 Plasma Sources Sci. Technol. $16 \mathrm{~S} 1$

[30] Robson R E, White R D and Ness K F 2011 J. Chem. Phys. 134064319

[31] Ness K F, Robson R E, Brunger M J and White R D $2012 \mathrm{~J}$. Chem. Phys. 136024318

[32] de Urquijo J, Basurto E, Juárez A M, Ness K F, Robson R E, Brunger M J and White R D 2014 J. Chem. Phys. 141 014308

[33] Pitchford L C et al 2017 Plasma Proc. Polym. 141600098

[34] Jovanović J V, Stojanović V, Raspopović Z, de Urquijo J and Petrović Z Lj 2019 Plasma Sources Sci. Technol. 28 045006

[35] Grofulović M, Alves L L and Guerra V 2016 J. Phys. D: Appl. Phys. 49395207

[36] Schmidt B, Berkhan K, Götz B and Müller M 1994 Phys. Scr. T53 30

[37] Nakamura Y and Kurachi M 1988 J. Phys. D: Appl. Phys. 21 718

[38] Morgan W L 1992 Plasma Chem. Plasma Process. 12449

[39] Gibson D K 1970 Aust. J. Phys. 23683

[40] Davies D K, Kline L E and Bies W E 1989 J. Appl. Phys. 65 3311

[41] Rabie M, Haefliger P, Chachereau A and Franck C M $2015 \mathrm{~J}$. Phys. D: Appl. Phys. 48075201

[42] Mirić J, Simonović I, Petrović Z Lj, White R D and Dujko S 2017 Eur. Phys. J. D 71289

[43] Zawadzki M, Chachereau A, Kočišek J, Franck C M and Fedor J 2018 J. Chem. Phys. 149204305

[44] Petrović Z Lj, Dujko S, Marić D, Malović G, Nikitović Ž, Šašić O, Jovanović J, Stojanović V and Radmilović-Radenović M 2009 J. Phys. D: Appl. Phys. 42194002

[45] Crompton R W 1967 J. Appl. Phys. 384093

[46] Thomas W R L 1969 J. Phys. B: At. Mol. Phys. 2551

[47] Tagashira H, Sakai Y and Sakamoto S 1977 J. Phys. D: Appl. Phys. 101051

[48] Sakai Y, Tagashira H and Sakamoto S 1977 J. Phys. D: Appl. Phys. 101035

[49] Boeuf J P and Marode E 1984 J. Phys. D: Appl. Phys. 171133

[50] Blevin H A and Fletcher J 1984 Aust. J. Phys. 37593

[51] Robson R E 1986 J. Chem. Phys. 854486

[52] Kondo K and Tagashira H 1990 J. Phys. D: Appl. Phys. 231175

[53] Robson R E 1991 Aust. J. Phys. 44685

[54] Robson R E 1995 Aust. J. Phys. 48677

[55] Nakamura Y 2007 Proc. 28th ICPIG (Prague) pp 224-6

[56] Dujko S, White R D and Petrović Z Lj 2008 J. Phys. D: Appl. Phys. 41245205

[57] Robson R E, White R D and Hildebrandt M 2017 Fundamentals of Charged Particle Transport in Gases and Condensed Matter (Boca Raton, FL: CRC Press)

[58] Dahl D A, Teich T H and Franck C M 2012 J. Phys. D: Appl. Phys. 45485201

[59] Dahl D A and Franck C M 2013 J. Phys. D: Appl. Phys. 46 445202

[60] Chachereau A, Rabie M and Franck C M 2016 Plasma Sources Sci. Technol. 25045005

[61] Chachereau A, Fedor J, Janečková R, Kočišek J, Rabie M and Franck C M 2016 J. Phys. D: Appl. Phys. 49375201

[62] Haefliger P, Hösl A and Franck C M 2018 J. Phys. D: Appl. Phys. 51355201

[63] Haefliger P and Franck C M 2018 Rev. Sci. Instrum. 89023114

[64] Pachin J, Hösl A and Franck C M 2019 J. Phys. D: Appl. Phys. 52235204

[65] Ridenti M A, Vivaldini T C, Lima I B and Pascholati P R 2010 AIP Conf. Proc. (AIP) vol 1245 pp 92-7

[66] Aschwanden T 1985 Die ermittlung physikalischer entladungsparameter in isoliergasen und isoliergasgemischen 
mit einer verbesserten Swarm-methode PhD Thesis ETH Zurich

[67] Phelps A V and Pitchford L C 1985 Phys. Rev. A 312932

[68] Ruíz-Vargas G, Yousfi M and de Urquijo J 2010 J. Phys. D: Appl. Phys. $\mathbf{4 3} 455201$

[69] Korolov I, Vass M, Bastykova N K and Donkó Z 2016 Rev. Sci. Instrum. 87063102

[70] Donkó Z, Hartmann P, Korolov I, Jeges V, Bošnjaković D and Dujko S 2019 Plasma Sources Sci. Technol. 28095007

[71] Pinhão N R, Loffhagen D, Vass M, Hartmann P, Korolov I, Dujko S, Bošnjaković D and Donkó Z 2020 Plasma Sources Sci. Technol. 29045009

[72] Hasegawa H, Date H and Shimozuma M 2007 J. Phys. D: Appl. Phys. 402495

[73] Robson R E 1985 Phys. Rev. A 313492

[74] Philippa B, White R D and Robson R E 2011 Phys. Rev. E 84 041138

[75] Kumar K, Skullerud H R and Robson R E 1980 Aust. J. Phys. 33343

[76] Stokes P W, Simonović I, Philippa B, Cocks D G, Dujko S and White R D 2018 Sci. Rep. 81

[77] Robson R E and Ness K F 1986 Phys. Rev. A 332068

[78] White R D, Ness K F and Robson R E 2002 Appl. Surf. Sci. 19226

[79] de Urquijo J, Arriaga C A, Cisneros C and Alvarez I $1999 \mathrm{~J}$. Phys. D: Appl. Phys. 3241

[80] Basurto E, Hernández-Ávila J L, Juárez A M and de Urquijo J 2013 J. Phys. D: Appl. Phys. 46355207

[81] de Urquijo J, Juarez A M, Rodríguez-Luna J C and RamosSalas J S 2007 IEEE Trans. Plasma Sci. 351204

[82] Šašić O, de Urquijo J, Juárez A M, Dupljanin S, Jovanović J, Hernández-Ávila J L, Basurto E and Petrović Z Lj 2010 Plasma Sources Sci. Technol. 19034003

[83] Bekstein A, de Urquijo J, Ducasse O, Rodríguez-Luna J C and Juárez A M 2012 J. Phys.: Conf. Ser. 370012006

[84] de Urquijo J, Alvarez I, Basurto E and Cisneros C 1999 J. Phys. D: Appl. Phys. 321646

[85] Datskos P G, Carter J G and Christophorou L G 1992 J. Appl. Phys. $\mathbf{7 1} 15$

[86] Simonović I, Bošnjaković D, Petrović Z Lj, White R D and Dujko S 2020 Eur. Phys. J. D 741

[87] Simonović I, Bošnjaković D, Petrović Z Lj, Stokes P W, White R D and Dujko S 2020 Phys. Rev. E 101023203

[88] Boyle G J, Tattersall W J, Cocks D G, Dujko S and White R D 2015 Phys. Rev. A 91052710
[89] Boyle G J, McEachran R P, Cocks D G and White R D 2015 J. Chem. Phys. 142154507

[90] de Urquijo J, Casey M J E, Serkovic-Loli L N, Cocks D G, Boyle G J, Jones D B, Brunger M J and White R D 2019 J. Chem. Phys. 151054309

[91] Hernández-Ávila J L, Basurto E and de Urquijo J 2004 J. Phys. D: Appl. Phys. 373088

[92] Hernández-Ávila J L, Basurto E and de Urquijo J 2004 UNAM database http://lxcat.net accessed 03 January 2018

[93] Kücükarpaci H N and Lucas J 1981 J. Phys. D: Appl. Phys. 14 2001

[94] Kücükarpaci H N and Lucas J 1981 IST-Lisbon database http:// lxcat.net accessed 03 January 2019

[95] Biagi S F Magboltz version 8.97 http://magboltz.web.cern.ch/ magboltz/ accessed 31 December 2017

[96] Haefliger P and Franck C M 2018 ETHZ (ETH Zurich, high voltage laboratory) database http://lxcat.net accessed 03 January 2019

[97] Foster S 2018 Self-consistency of electron-THFA gaseous cross-sections via Swarm techniques Undergraduate Honors Thesis James Cook University

[98] Xiao D M, Zhu L L and Chen Y Z 1999 J. Phys. D: Appl. Phys. $32 \mathrm{~L} 18$

[99] Nakamura Y 1988 J. Phys. D: Appl. Phys. 2167

[100] Naidu M S and Prasad A N 1972 J. Phys. D: Appl. Phys. 5 1090

[101] Biagi S F 2014 Biagi database (Magboltz version 10.6) http:// lxcat.net/Biagi accessed 03 January 2019

[102] Christophorou L G and Olthoff J K 2000 J. Phys. Chem. Ref. Data 29267

[103] de Urquijo J, Basurto E and Hernández-Ávila J L 2001 J. Phys. D: Appl. Phys. 342151

[104] Xiao D M, Li X G and Xu X 2001 J. Phys. D: Appl. Phys. 34 L133

[105] Petrović Z Lj, Marić D, Savić M, Marjanović S, Dujko S and Malović G 2017 Plasma Proc. Polym. 141600124

[106] Crompton R W and Jory R L 1962 Aust. J. Phys. 15451

[107] Crompton R W, Elford M T and Gascoigne J 1965 Aust. J. Phys. 18409

[108] Elford M T 1971 Aust. J. Phys. 24705

[109] Crompton R W and Elford M T 1973 Aust. J. Phys. 26771

[110] Robson R E, White R D and Petrović Z Lj 2005 Rev. Mod. Phys. 771303

[111] Mladenović Ž, Gocić S, Marić D and Petrović Z Lj 2018 Eur. Phys. J. Plus 133344 